\title{
Quantum information erasure inside black holes
}

\author{
David A. Lowe ${ }^{a}$ and Larus Thorlacius ${ }^{b, c}$ \\ ${ }^{a}$ Department of Physics, Brown University, \\ Providence, RI, 02912, U.S.A. \\ ${ }^{b}$ University of Iceland, Science Institute, \\ Dunhaga 3, IS-107 Reykjavik, Iceland \\ ${ }^{c}$ The Oskar Klein Centre for Cosmoparticle Physics, Department of Physics, \\ Stockholm University, AlbaNova University Centre, 10691 Stockholm, Sweden \\ E-mail: lowe@brown.edu, 1th@hi.is
}

ABSTRACT: An effective field theory for infalling observers in the vicinity of a quasi-static black hole is given in terms of a freely falling lattice discretization. The lattice model successfully reproduces the thermal spectrum of outgoing Hawking radiation, as was shown by Corley and Jacobson, but can also be used to model observations made by a typical low-energy observer who enters the black hole in free fall at a prescribed time. The explicit short distance cutoff ensures that, from the viewpoint of the infalling observer, any quantum information that entered the black hole more than a scrambling time earlier has been erased by the black hole singularity. This property, combined with the requirement that outside observers need at least of order the scrambling time to extract quantum information from the black hole, ensures that a typical infalling observer does not encounter drama upon crossing the black hole horizon in a theory where black hole information is preserved for asymptotic observers.

KeYwords: AdS-CFT Correspondence, Models of Quantum Gravity, Black Holes

ArXiv EPrint: 1508.06572 


\section{Contents}

1 Introduction 1

2 Free falling lattice model $\quad 2$

$\begin{array}{lll}3 & \text { Dispersive propagation } & 7\end{array}$

$\begin{array}{lll}4 & \text { Number operators } & 11\end{array}$

$\begin{array}{lll}5 & \text { Equivalence principle violations } & 13\end{array}$

6 Conclusions 14

\section{Introduction}

Recently the authors proposed a method to build quasi-local bulk operators inside black hole horizons in unitary quantum theories [1]. The method relies on two conjectured properties of such theories:

1. that the minimal decoherence time of a black hole (or the time required for outside observers to extract quantum information from the black hole) has a lower bound of order the scrambling time, $t_{\mathrm{scr}}=4 M \log (4 M),{ }^{1}$

2. that, from the viewpoint of an infalling observer who enters the black hole, any quantum information that entered more than a scrambling time earlier has been erased.

The construction in [1] is tailored to fit observations made by an observer falling into a black hole at a prescribed time and is only valid for a scrambling time before and after the observer enters the horizon. During this restricted time period, the resulting effective theory describes observations made by a typical low-energy infalling observer, to within the limited experimental precision available to such an observer, and its predictions agree with those the underlying unitary theory to well within the observable precision.

In the present paper we consider a simple discretization of the radial direction in an infalling reference frame and explore the consequences for the construction of quasi-local bulk operators in the black hole interior. This can be viewed as a concrete realization, with an explicit short distance cutoff, of the effective field theory construction in [1]. The same type of discretization has been considered before in the work of Corley and Jacobson [2], in a dimensionally reduced model for black hole evaporation. There the focus was on the black hole exterior and showing that the spectrum of the Hawking flux is insensitive to

\footnotetext{
${ }^{1}$ More generally $t_{\mathrm{scr}}=\mathcal{O}(\beta \log S)$, with $\beta$ the inverse Hawking temperature and $S$ the BekensteinHawking entropy.
} 
the discretization. It turns out the model is also suitable for a bulk effective description of low-energy observers who enter a black hole in free fall. As we shall see below, the combination of large redshift factors in the black hole region and having a strict short distance cutoff severely restricts the amount of quantum information accessible to a typical infalling observer inside the black hole. Combined with property \#1, this ensures that a typical infalling observer does not encounter drama upon crossing the black hole horizon [1].

General covariance is the symmetry principle underlying Einstein's theory of gravitation. The essence of the black hole information problem, is that in a quantum theory, general covariance leads to a conflict between unitarity and bulk locality. Holographic models, such as the anti-de Sitter gravity/conformal field theory (AdS/CFT) correspondence, provide a setting where the unitarity puzzle is resolved at the expense of bulk locality. It is less well-understood, however, how the violation of bulk locality avoids infecting observations made by low-energy local observers both inside and outside the black hole in the limit where one expects the semiclassical approximation for bulk gravity to be good. We note that a finite $N$ reconstruction of the bulk geometry from the holographic theory involves a soft violation of general covariance. The symmetry is expected to be restored in the $N \rightarrow \infty$ limit but Hawking emission is a $1 / N$ effect in holographic theories and the black hole information problem cannot be studied in the strict $N \rightarrow \infty$ limit. In AdS/CFT examples, symmetries associated with the transverse directions are linearly realized in the holographic description, and such directions are reconstructed exactly in the bulk theory. However, the bulk radial direction arises from scale transformations, which are non-linearly realized in the quantum holographic theory, and the breaking of general covariance is implemented through a subtle discretization of the radial direction. The details of this holographic regulator are currently poorly understood, in particular when the bulk spacetime contains a black hole.

There is general consensus that quasi-local bulk operators can be constructed in black hole exteriors on length scales approaching the Planck length. In particular, the bulk effective Hamiltonian can be constructed for a quantum field in the background of a large black hole [3]. One of the central ideas behind the construction of [1], was that evolution of exterior operators under this Hamiltonian provides a natural definition of interior bulk operators. The discretization we consider here provides a model where the properties of such interior operators may be explored. The description is only approximate and does not capture the holographic physics in all detail. However, this is not a problem since observations made by an inside observer in free fall necessarily have limited precision both due to the restricted size of measuring devices that can be carried into a black hole without back-reacting on the geometry and due to the finite proper time remaining to the observer before encountering the black hole singularity [4]. Any effective field theory or other model description for infalling observers need only reproduce the exact holographic physics to within this limited precision [4].

\section{$2 \quad$ Free falling lattice model}

In a black hole exterior, there seems to be considerable freedom in defining bulk operators in holographic models. This makes sense, because at least for arbitrarily large distances 
from the black hole, one can consider effective field theories with large relative boosts with respect to the black hole, without having to worry about issues of back-reaction. Here by effective field theory, we mean a field theory with a local Lagrangian, which may be accurately treated using a semiclassical expansion, ignoring quantum gravitational effects.

Close to the black hole horizon, this is in general no longer the case. One is not free to consider effective theory in an arbitrary frame where typical energies are trans-Planckian with respect to the black hole rest frame. Nevertheless, the equivalence principle dictates that we should be able to construct an effective field theory in a freely falling frame outside the black hole, where typical local frequencies are much smaller than the Planck scale. From the holographic perspective, this means we should be able to construct quasi-local operators, localized on scales $\lambda$ in this frame satisfying $G M \gg \lambda \gg l_{\text {Planck }}$, where $l_{\text {Planck }}$ is the Planck length.

Of course, one might argue that if the holographic theory is to truly describe generally covariant gravity, it must also accommodate observers or probes with arbitrarily large relative boosts with respect to the black hole. At the end of the day, such a requirement imposes general covariance on the quantum theory. It is clear, however, that the holographic theory does not contain general covariance as a manifest symmetry for finite $N$. Certainly in a large $N$ limit one hopes it is equivalent to classical string theory in the background in question, but to see the Hawking radiation in the first place, it is essential to keep $N$ (or more generally the central charge of the conformal field theory) finite. Our point of view will be that the holographic theory is able to supply an effective field theory in a coordinate patch satisfying the conditions of the previous paragraph, but is not fully generally covariant, and our task is to study whether such an effective field theory is sufficient to describe the physics of an infalling observer as they cross the horizon, where general covariance does emerge in a classical limit.

A two-dimensional free-falling lattice model was studied by Corley and Jacobson [2], where their main goal was to show that the spectrum of Hawking radiation was insensitive to the details of the short distance cutoff. In the present work, we use essentially the same model, treating the radial direction as a free falling lattice with Planck scale lattice spacing near the horizon. Rather than work with a two-dimensional model, one can also generalize to four dimensions, treating the angular directions as continuous spheres, but for our purposes it turns out to be sufficient to consider the s-wave sector. We will be focusing on near-horizon physics, so we restrict discussion to the simple case of a Schwarzschild black hole in asymptotically flat spacetime. Generalization to spacetimes with other asymptotics will not affect our main conclusions. Furthermore, since we will be considering timescales that are parametrically short compared to the black hole lifetime, we will model the background geometry as a static Schwarzschild spacetime.

The starting point for the lattice model is the Schwarzschild metric in GullstrandPainlevé coordinates (in units with $G=\hbar=c=1$ ). We define

$$
v(r)=-\sqrt{\frac{2 M}{r}},
$$

and then the metric is

$$
d s^{2}=-\left(1-v^{2}(r)\right) d t^{2}-2 v(r) d t d r+r^{2} d \Omega^{2},
$$


where the time coordinate is obtained by adding an $r$-dependent function to the usual Schwarzschild time

$$
t=t_{s}+2 M\left(-\frac{2}{v(r)}-\log \left(\frac{1-v(r)}{1+v(r)}\right)\right) .
$$

The coordinate transformation is singular on the horizon, as it must be for the GullstrandPainlevé coordinates to be smooth there. We note, however, that this is traded for a coordinate singularity at large $r$.

For the moment, we take the metric to be of the form (2.1) inside the horizon of the black hole $r<2 M$. This amounts to assuming that neither additional matter stress energy nor gravitational waves are present inside the horizon. This may appear to be rather a strong assumption given that the lattice model based on this metric is to be viewed as a representation of the exact physics described by the holographic theory. However, as we see later on (towards the end of section 3), there is a sense in which the metric (2.1) behaves as an attractor in the regulated theory.

The metric is stationary in Gullstrand-Painlevé coordinates so $p_{t}$ is conserved along a timelike geodesic. For a particle of unit mass on a radial geodesic we have

$$
p_{t}=-E=-\left(1-v^{2}(r)\right) \frac{d t}{d \tau}-v(r) \frac{d r}{d \tau}
$$

Imposing the usual normalization condition on the 4-velocity of the particle, leads to the orbit equation for radial geodesics

$$
\left(\frac{d r}{d \tau}\right)^{2}=E^{2}-1+v^{2}(r)
$$

The infall coordinates used in [2] are constructed from geodesics with $E^{2}=1$, which are at rest at infinity. For this choice, $t$ is equal to the proper time along the geodesic. One may then introduce a new radial coordinate

$$
y=t-\int_{2 M}^{r} \frac{d r^{\prime}}{v\left(r^{\prime}\right)},
$$

which is constant along the geodesic. In these coordinates, the metric is

$$
d s^{2}=-d t^{2}+v^{2}(r) d y^{2}+r^{2} d \Omega^{2}
$$

with

$$
r(y, t)=2 M\left(1+\frac{3}{4 M}(y-t)\right)^{2 / 3} .
$$

The horizon is located at $y=t$ and the curvature singularity at $y=t-\frac{4}{3} M$. In particular, the $y=0$ geodesic enters the horizon at $t=0$, and hits the singularity at $t=\frac{4}{3} M$.

The freely falling lattice model is obtained by discretizing the $y$ coordinate. We choose a freely falling Planck scale lattice near the horizon (rescaling $M$ can be used to rescale this to any desired length), as motivated by holographic models. At larger radius, the proper spacing falls below the Planck length, limiting the region of spacetime where the effective field theory description will be valid. However this will be sufficient for our purposes, and 
was already sufficient to show cutoff independence of the Hawking flux. The breakdown of the free-fall lattice regulator far from the black hole is in line with black hole complementarity. Presumably to represent the far region using a holographic description, one must evolve operators with respect to a different time coordinate, such as the Schwarzschild time, resulting in a very different regulator in the bulk effective field theory.

Let us consider a massless scalar field on the freely falling lattice. We choose units such that the lattice spacing in $y$ is 1 . Since the most dangerous modes for us are s-waves, it is convenient to truncate to only those modes. The Lagrangian is then

$$
S=2 \pi \sum_{y} \int d t r^{2}(y, t)\left(|v(r(y, t))|\left(\frac{\partial \phi}{\partial t}\right)^{2}-\frac{2\left(D_{y} \phi\right)^{2}}{|v(r(y+1, t))+v(r(y, t))|}\right),
$$

with the difference operator

$$
D_{y} \phi=\phi(y+1, t)-\phi(y, t) .
$$

An attractive feature of this discretization is that the action has a residue of the Killing symmetry of the Schwarzschild metric

$$
(y, t) \rightarrow(y+1, t+1) .
$$

In particular, this allows one to use separation of variables to derive the form of the mode functions, and determine the lattice dispersion relation. The general form of the mode function takes the form

$$
\phi(y, t)=e^{-i \omega t} f(y-t),
$$

where the frequency $\omega$ may be taken to be any real number. The invariance of the mode function under

$$
k \rightarrow k+2 n \pi, \quad \omega \rightarrow \omega-2 n \pi
$$

for any integer $n$ allows one to map an arbitrary pair $(\omega, k)$ into the range

$$
\begin{gathered}
-\pi<k \leq \pi \\
-\infty<\omega<\infty .
\end{gathered}
$$

In a WKB approximation, we expand the mode function in terms of wavevectors that depend on position

$$
\phi(y, t)=e^{-i \omega t} e^{i k(r)(y-t)} .
$$

Plugging (2.9) into the equation of motion, and assuming $r(y, t)$ varies slowly on the length scale defined by the local wave vector, yields the dispersion relation obtained by Corley and Jacobson [2] for the WKB wavevector $k(r(y, t))$

$$
|v(r)|(\omega+k)= \pm 2 \sin (k / 2) .
$$

This dispersion relation governs the propagation of wavepackets in the $(y, t)$ plane. It can be solved numerically but the qualitative behavior of solutions can be obtained by simple 


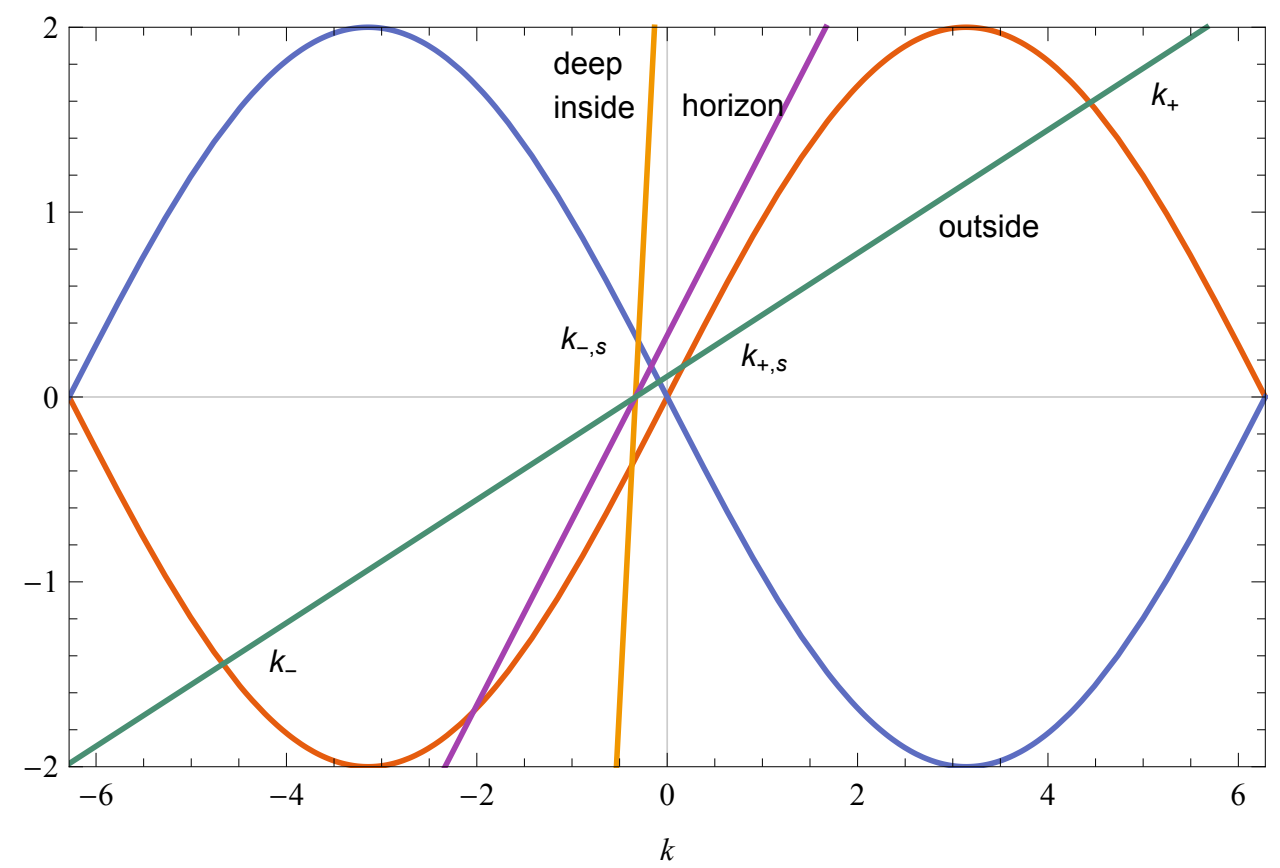

Figure 1. Graphical solution of the lattice dispersion relation. The straight lines correspond to the left hand side of (2.10) for different values of $v(r)$ (and $\omega>0$ ).

graphical analysis based on figure 1 . The straight lines in the figure correspond to the lefthand side of (2.10) for $\omega>0$ and three different values of $r$; outside, inside and at the black hole horizon, with the slope of the line determined by $v(r)$ in each case. Far away from the black hole $v(r) \rightarrow 0$ and the line approaches horizontal, while deep inside the black hole it becomes vertical. For a given value of $r$, the solutions to the dispersion relation are given by the points where the corresponding straight line intersects the sinusoidal curves. The sign of the group velocity in the $y$ coordinate

$$
\frac{d y}{d t}= \pm \frac{1}{|v(r)|} \cos (k / 2)
$$

determines whether the mode is right- or left-moving. For each $\omega>0$ solution represented in figure 1, there is a corresponding one with $\omega<0$ (and opposite sign $k$ ). The two branches of solutions are related by complex conjugation and we will restrict attention to the $\omega>0$ case.

Far away from the black hole there are four intersection points, corresponding to four different $\omega>0$ modes, of which two survive the continuum limit and two are only present at finite lattice cutoff. Following [2], we denote the modes by $\psi_{+}, \psi_{-}, \psi_{+, s}$, and $\psi_{-, s}$, and let the corresponding WKB momenta carry the same subscripts, as indicated in figure 1. Of these modes, $\psi_{-, s}$, is left-moving while the other three are right-moving. The momenta $k_{+}$and $k_{-}$lie outside the fundamental domain in (2.8) but can be brought inside it by a shift of the form (2.7).

Free fall observers, traveling along lines of constant $y$ measure the free-fall frequency

$$
\omega_{\mathrm{ff}}=\omega+k,
$$


which is invariant under the shift symmetry (2.7). In particular, low-energy observers in free fall will only have access to modes where $\left|\omega_{\mathrm{ff}}\right| \ll 1$. Note that a $\omega>0$ mode can have negative free-fall frequency. This applies, for instance, to the $k_{-}$mode in figure 1.

As we move towards the black hole, the intersection points migrate along the sinusoidal curves and at a critical value of $r$ the two right-moving modes with positive $k$ merge, leading to a WKB turning point outside the black hole [2]. At smaller values of $r$ only two $\omega>0$ modes remain: $\psi_{-}$, which is right-moving, and $\psi_{-, s}$, which is left-moving.

It is worth commenting that the lattice gives rise to a continuum of modes in $(\omega, k)$ space, which include wavepackets localized behind the horizon. Presumably in the exact quantum theory there is some further discretization leading to a finite number of states behind the black hole horizon. It is reasonable this should be invisible in any semiclassical approximation since the expected energy level spacing will be of order $e^{-S_{b h}}$ where $S_{b h}$ is the Bekenstein-Hawking entropy. Certainly such a refined level spacing is irrelevant to infalling observers behind the horizon, who only live for a maximal proper time of order the light-crossing time of the black hole.

\section{Dispersive propagation}

The bulk Hamiltonian in these coordinates generates evolution along timelike geodesics from outside the horizon all the way to the singularity. Unitarity is manifest, except for the component of the wavefunction that hits the spacelike singularity. In effect, the collision of points with the singularity replaces the curtains of fire suggested by some authors ${ }^{2}[5,6]$. The trick is to come up with a self-consistent way to maintain the validity of the interior freely falling description, while having a complementary unitary exterior evolution. As discussed in detail in [1], this can be accomplished in theories of quantum gravity that obey properties \#1 and \#2 listed in the Introduction. Our immediate goal is to derive property \#2 within the lattice model of the previous section. This provides a concrete example of the behavior argued for on general grounds in our earlier work [1] and lends credence to the self-consistency of our general effective field theory picture for infalling observers put forward in that work.

We wish to estimate the correlation function of a wavepacket operator, specified to be inside the horizon on a given time slice, with another similarly constructed wavepacket evolved by free fall from outside the horizon, crossing the horizon at a later time. The by now standard theory of decoherence (for a textbook treatment see [7]) shows that local interactions with a large number of exterior degrees of freedom decohere a quantum state, if given sufficient time. In the case of a black hole this decoherence time is at least of order the scrambling time [8]. Correlation functions between wavepackets that enter the horizon separated by less than a scrambling time are not affected by decoherence. However, if our initial wavepacket is capable of sending a detectable signal to a late time wavepacket that enters the black hole a scrambling time or more later, then the black hole complementarity

\footnotetext{
${ }^{2}$ At the singularity one needs a prescription to evolve the state forward in time, in order to preserve its normalization. A natural prescription is to trace over modes that hit the singularity, mimicking how one treats the exterior region in the original proposal of Hawking.
} 

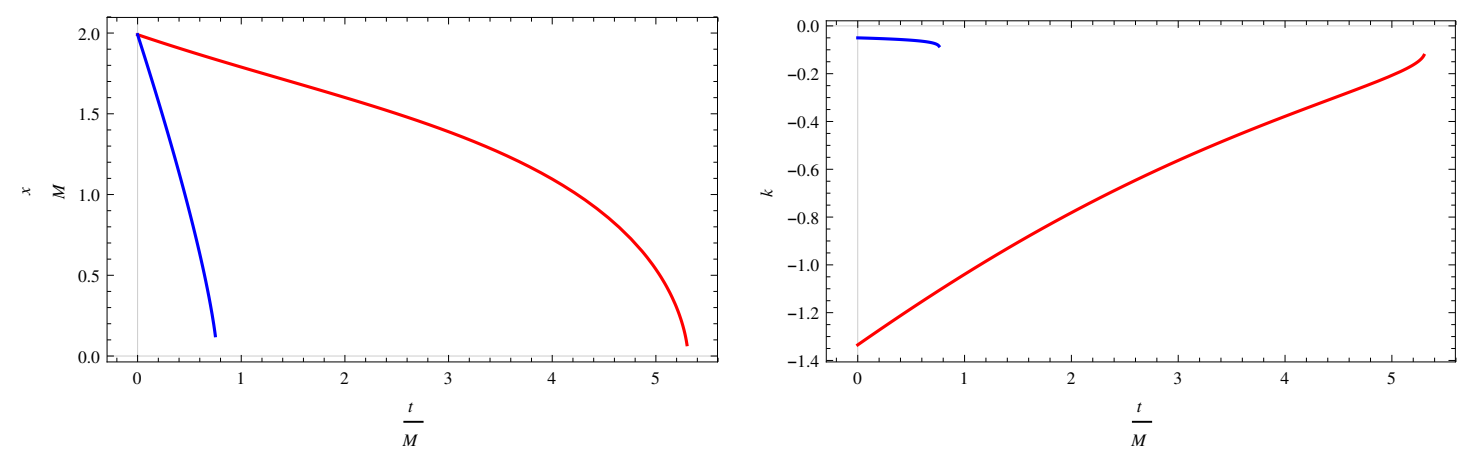

Figure 2. Interior wavepacket trajectories. The left figure shows the radial position as a function of the Gulstrand-Painlevé coordinate $t$. The right figure shows the evolution of the WKB wavevector $k$. The red curves represent the right-moving, horizon skimming mode, which take a scrambling time to hit the singularity. The blue curves represent the left-moving mode. In the figures, $M=$ $100, \omega=1 / 10$.

principle will be falsified [9]. In other words, if one takes property \#1 as a postulate, then a violation of property \#2 implies violation of black hole complementarity.

It is straightforward to analyze the propagation of such wavepackets, using standard methods of propagation in dispersive media [10]. The largest amplitude component of the wavepacket propagates within a timelike cone, bounded by the evolution of trajectories according to the group velocity of the different Fourier components. The group velocity with respect to the $y-t$ coordinate of a wavetrain (2.6) is

$$
v_{g}=\frac{d \omega}{d k}= \pm \frac{\cos (k / 2)}{|v|}-1
$$

Let us begin with a wavepacket just inside the horizon. This is inside the future trapped region so both signs in (3.1) correspond to ingoing wavepackets. The minus sign gives rise to a left-moving wavepacket, i.e. one that moves towards negative $y$ in the infalling lattice, while the plus sign gives a right-moving wavepacket, moving towards positive $y$. The plot on the left in figure 2 shows numerical results for the radial position of left- and right-moving wavepackets as a function of time, while the plot on the right tracks the value of the wavevector $k_{\max }$, where the wavepacket has maximum amplitude. The left-moving mode rapidly hits the singularity, as expected, with little change in $k_{\max }$. The right-moving horizon-skimming modes are of more interest to us as they take much longer to hit the singularity and the WKB value for $k_{\max }$ can evolve significantly along the way.

In figure 2 the right-moving wavepacket starts out near the horizon with a large wavevector so the free fall frequency $\omega_{\mathrm{ff}}$ of this mode is large compared to $\omega$. In view of this, it is instructive to compare the free fall frequencies of left- and right-moving wavepackets near the horizon in the limit $\omega \ll 1$. Inserting $v=-1$ into (2.10) and solving for small $|k|$ gives $\omega_{\mathrm{ff}} \approx-k$ for left-moving modes, which is of the same order of magnitude as $\omega$. For a right-moving wavepacket one instead finds

$$
\omega_{\mathrm{ff}} \approx-(24 \omega)^{1 / 3}
$$


The appearance of this intermediate scale in the regulated theory was noted in [2], though in that case the focus was on exterior modes, where the same $\omega^{1 / 3}$ behavior is seen near the classical turning point in the WKB approximation. The existence of such an intermediate length scale in the regulated theory may be of interest in the context of the non-local information transfer across the event horizon proposed in [11].

Inside the horizon, as is clear from figure 1, there is no classical turning point, so a straightforward WKB approximation should be valid for interior wavepackets until they approach the curvature singularity. Let us consider the correlator of a right-moving interior

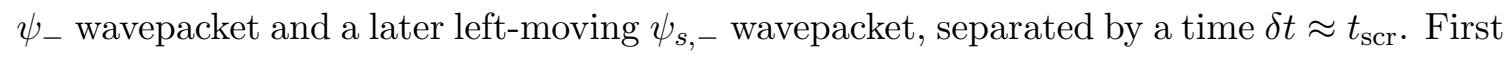
we estimate the width of the $\psi_{-}$wavepacket, by the time it has propagated to the late time slice. To proceed, let us begin with a wavepacket of the form

$$
\psi_{\text {initial }}(y, t)=\int d \omega e^{-\left(\omega-\omega_{0}\right)^{2} a^{2}} e^{i k_{-}(\omega, r)(y-t+d)-i \omega t},
$$

where $d \gg a>1$ and $\omega_{0} \sim 1 / a$ in order that the wavepacket be well-localized at $y=-d$ inside the horizon at $t=0$. The dominant components of the wavepacket travel at the group velocity (3.1) (with + sign) which is less than the speed of light with respect to the infalling lattice. The time it takes the dominant part of the wavepacket to hit the singularity is therefore bounded from above by the time it takes a right-moving radial null geodesic to hit the singularity starting from the an initial point $(y, t)=(-1,0)$ just inside the horizon. On such a geodesic

$$
\frac{d y}{d t}=-\frac{1}{v(r)}
$$

Using (2.3) one obtains

$$
\frac{d r}{d t}=1+v(r)
$$

which integrates to

$$
\frac{t}{4 M}=\frac{r}{4 M}-\frac{1}{v(r)}+\log \left|1+\frac{1}{v(r)}\right|+A
$$

where $A$ is an integration constant to be determined by the condition it pass through the initial point. For large $M$

$$
A \approx \log (4 M)-\frac{3}{2}+\mathcal{O}(1 / M) .
$$

The geodesic hits the singularity at

$$
t=4 M A \approx 4 M \log 4 M,
$$

which we define as the scrambling time $t_{\mathrm{scr}}$.

Returning to our initial right-moving wavepacket, we see from this that it will hit the singularity no later than at the scrambling time. As the singularity is approached the metric diverges, $|v(r)| \rightarrow \infty$, in which case the dispersion relation (2.10) reduces to $\omega=-k$ and the width in $y$ of the wavepacket becomes of order $a$. After a scrambling time the singlularity is located at $y \approx t_{\mathrm{scr}}$, and it follows that the overlap of the initial 
wavepacket with another incoming wavepacket at the horizon is at most of order $e^{-t_{\mathrm{scr}}^{2} / a^{2}}$ in the WKB approximation.

In addition to the dominant components of the wavepacket that travel at the group velocity, there are the so-called forerunners [10]. These are modes with different frequencies than the dominant component of the signal and can travel faster than the dominant group velocity. Such modes are substantially excited only when the group velocity has a maximum or minimum, which is when the straight lines in figure 1 are tangent to the sine curves. The only such turning point for the modes appears outside the horizon, so this is not relevant for the interior propagation. In the absence of a WKB turning point, the forerunners of an interior wavepacket form a tail of low-frequency modes with $k_{-} \approx-(24 \omega)^{1 / 3}$. This tail essentially corresponds to the part of the initial wavepacket that is outside the horizon at the initial time and its size can be estimated by evaluating the integral in (3.3) in a saddle point approximation. The resulting contribution to the overlap with the late time wavepacket is of order $e^{-c d^{2} / a^{2 / 3}}$, where $c$ is an order one numerical constant, and is strongly suppressed when $d \gg a>1$.

Thus far we have simply assumed an interior vacuum spacetime using the metric (2.1) inside the horizon. However we have concluded that both massive and massless matter excitations inside the horizon hit the singularity within a scrambling time in the lattice model. The same will be true for metric perturbations treated as background fields in a lattice model and we conclude that the interior metric effectively relaxes back to the vacuum within a scrambling time. Therefore from the viewpoint of the holographic theory, assuming the metric (2.1) should be reasonable as long as the amount of matter that enters the black hole during a scrambling time prior is insufficient to cause significant back-reaction on the geometry.

As a potential counterexample, consider a right-moving negative energy shockwave crossing the path of an infalling observer. This can cause the $M$ parameter of (2.1) to decrease by an arbitrarily large amount (after performing a suitable change of coordinates). This would invalidate the conclusions of the previous section, where it was argued the dispersion relation leads to information erasure within a scrambling time. This is an extreme example of the so-called "pots of gold" solutions obtained by patching across domain walls of stress energy [12-14]. If one imposes reasonable energy conditions on the domain walls, however, these dangerous classical solutions are removed.

One might still worry about the effect of back-reaction of the Hawking emission itself on the interior metric. However a number of Hawking particles of order $\log S$ are emitted during a scrambling time, and their typical energies are of order the Hawking temperature. Therefore we do not expect a large effect on the interior metric since our patch is only to be used for a range of times of order the scrambling time.

A detectable late-time signal in the interior would require an enormous amplitude, either for the detector or the emitter, or both. Due to the enormous exponential suppression factor, in either case, the required trans-Planckian energy of the detector or emitter would produce enormous back-reaction on the black hole. We conclude therefore, that for lowenergy observers, quantum information is effectively erased in the black hole interior after a scrambling time has passed. This establishes the validity of property \#2 in this model. 


\section{Number operators}

One immediate cause for concern is that virtual modes with relatively large free fall frequencies such as (3.2) will cause apparent violations of general covariance for a freely falling timelike observer, due to their large free fall frequency, despite having a small frequency $\omega$ with respect to the timelike Killing vector.

Outside the horizon, there are four solutions to the dispersion relation: $\psi_{+}, \psi_{-}, \psi_{+, s}$ and $\psi_{-, s}$ (in fact even more solutions may appear at very large $r$ ). The corresponding wavevectors are indicated in figure 1. A central result of [2] is that the low-energy latetime outgoing mode $\psi_{+, s}$ is entangled with the modes $\psi_{-}$and $\psi_{+}$if one imposes a free fall vacuum state far from the horizon. In particular, the occupation number of the late-time $\psi_{+, s}$ modes is simply a Bose-Einstein distribution with respect to the frequency $\omega$, thus the lattice model reproduces the expected Hawking spectrum

$$
N_{\omega}=\frac{1}{e^{\omega / k T}-1}
$$

where $T$ is the Hawking temperature of the black hole.

For the present work, it is also interesting to consider the number operators associated with other wavepackets of interest. Some formalism helpful in setting up these questions appears in [15]. The easiest modes to consider are the $\psi_{-, s}$ modes. These correspond to ingoing modes that freely propagate all the way to the singularity. Because the WKB approximation is valid all the way along their trajectory, there is negligible scattering into other modes. Hence these modes have no effect on the late-time outgoing mode $\psi_{+, s}$. Their free fall frequency is positive, so they are annihilated by the initial free fall vacuum. The number operator $N_{-, s}$ will therefore have vanishing expectation value in the free fall vacuum.

Now consider the large wavevector mode $\psi_{+}$. This mode has positive free fall frequency, so at early times is annihilated by the free fall vacuum. At late times, the mode propagates to a classical turning point outside the horizon, where it undergoes mode conversion to the outgoing mode $\psi_{+, s}$ and also tunnels into the interior. Wavepackets corresponding to this mode can be constructed prior to the mode hitting the classical turning point. Clearly evolving these back in time, the WKB approximation remains valid, and the associated number operator will have vanishing expectation value. Wavepackets after the turning point correspond to the outgoing mode $\psi_{+, s}$ that we discussed above.

Finally, let us consider the large wavevector mode $\psi_{-}$. This has negative free fall frequency, so the annihilation operator corresponds instead to the conjugate mode $\psi_{-}^{*}$. It is possible to build wavepackets dominated by the $\psi_{-}^{*}$ mode. In the continuum limit, these modes would be localized inside the horizon (as is easily seen by replacing the sine curves in 1 by straight lines). However with the regulator, the modes also have support outside the horizon. This is a crucial new feature of the regulated theory. Building a wavepacket well-approximated by such a mode will evolve across the horizon and continue toward the singularity, again with the WKB approximation remaining valid. We conclude that the number operator of such a wavepacket will have vanishing expectation value, since it can 
be smoothly propagated back to the initial timeslice where the $\psi_{-}^{*}$ mode annihilates the free fall vacuum.

It is worth reiterating that while a Fock space built using the WKB modes appears larger than the Fock space of the continuum theory, one is not adding extra degrees of freedom. The action for the fields (2.4) remains that of a local scalar field with a lattice regulator.

It is interesting to compare these results with the conclusions of [16], who argued that if the infalling number operators were built out of exterior operators, a firewall would result. An alternative proposal for building interior operators for old black holes was proposed in $[17,18]$, by embedding the exact holographic state in a larger semiclassical Hilbert space using ancillae modes set in their ground state. In the lattice model presently considered we see the ancillae directly arise in the exterior region in the form of the WKB modes $\psi_{+}$ and $\psi_{-}$, providing a concrete realization of the ideas of [18]. If the lattice model correctly captures the relevant physics of the holographic theory, then we see that ancillae modes arise, and that their initial state is determined by the exterior state of the black hole via their free fall vacuum state. In the continuum limit, the mode $\psi_{+}$is never present, and the mode $\psi_{-}$has support only inside the black hole horizon, rather than at arbitrarily large distance from the horizon as in the lattice model.

The $\psi_{-}$is the dangerous mode from the point of view of the information problem. If not correctly entangled with the outgoing mode $\psi_{+, s}$ via the free fall vacuum condition, the expectation value of the stress energy tensor will be large. We note in the regulated theory, this is even true well outside the horizon. In the continuum limit the issue appears only across the horizon leading to the so-called firewall proposal $[5,6]$.

The regulated effective description described in the present work is only valid inside and near the horizon, and is suitable only for the approximate representation of observations by an infalling observer, who necessarily has a finite lifetime, and corresponding finite experimental uncertainties. The regulated effective description gives rise to Hawking radiation with the usual thermal spectrum [2], but it imparts no information about the black hole state Hawking radiation. As emphasized in [1], this restricts its usefulness to a limited range in time extending a scrambling time before and after the infalling observer enters the black hole, but this range is sufficient to correctly describe observations made by the infalling observer to within the available experimental precision.

A complementary effective field theory description will be valid for purely exterior observers whose observations must coincide with the exact unitary description of the black hole evolution, but this requires cutting out a timelike stretched horizon, where the boundary conditions can no longer be represented by an ordinary local effective field theory. It also requires using a regulator that smoothly extends to infinity and is naturally formulated on the usual Schwarzschild coordinate patch rather than the infalling Gullstrand-Painlevé coordinates which break down in the asymptotic region.

If on the other hand, we had made the inconsistent assumption, that is the foundation of the firewall argument, that a single effective field theory description allows one to describe the black hole interior and the exact outgoing Hawking radiation, then the $\psi_{\text {- mode would }}$ necessarily not be entangled with $\psi_{+, s}$. In that case, there would be a firewall inside the 
horizon in the continuum theory and introducing a lattice regulator would lead to the firewall spreading outside the horizon into the exterior region, since information retrieval would be incompatible with the initial free fall vacuum.

\section{Equivalence principle violations}

One of the main conclusions of the present work and that of [2] is that it is very difficult to construct experiments that detect violations of general covariance. Hawking radiation appears to emerge with a largely undistorted spectrum, and experiments in the vicinity of black holes seem compatible with general covariance, as long as Planck scale accelerations are not invoked.

It is of interest then to see if the lattice model, which arguably represents the behavior of a wide class of holographic theories, offers any distinctive signals of the violation of general covariance. The simplest experiment to construct simply involves scattering a wavepacket far from the black hole, off the horizon, to be observed later. This involves the same mode function computations as in [2], the difference being we now consider a real quantum wavepacket scattering, rather than a virtual one. Tracing back the late time signal through the turning point, and back out into the far region, we obtain a mode composed of the two WKB modes $\psi_{+}$and $\psi_{-}$corresponding to the large $|k|$ solutions of the dispersion relation (2.10) at large $r$. There will also be an unscattered component that we denote $\psi_{\text {early }}$ corresponding to the small $|k|$ solution of the dispersion relation. The creation operator for the late-time mode will take the form

$$
a_{\text {late }}^{\dagger}=a_{+}^{\dagger}+a_{-}
$$

when evolved back to the initial timeslice. The free-fall initial state is annihilated by the $a_{-}$operator. The interesting component is then the piece corresponding to $a_{+}^{\dagger}$.

This component of the wavepacket has the interesting property that it scatters directly off the black hole horizon, despite being an infalling mode. Ordinarily, the low $k$ mode with $k<0$ propagates to the singularity with negligible scattering off the geometry. Instead, we find if this large $k$ ingoing mode is excited, it propagates to a turning point outside the horizon, and does a combination of back-scattering out, and tunneling inside. This is a dramatic new feature of horizon scattering, that conceivably could give rise to detectable signatures.

To better understand this mode, we convert from the coordinate large $k$ to the proper wavevector in the far region. To do this we must shift $k \rightarrow k-2 \pi, \omega \rightarrow \omega+2 \pi$ according to the symmetry $(2.5)$ and use $k_{\text {proper }}=k /|v(r)|$. In the low $\omega$ limit, this gives $k_{\text {proper }}=$ $-2 \pi-\omega$ in units of the cutoff, and the proper frequency becomes $\omega+2 \pi$. If the cutoff is taken close to the Planck scale, this indicates $\psi_{+}$corresponds to a Planck energy mode.

Nature appears to naturally produce jets of highly energetic particles in the vicinity of rotating black holes. The model suggests that such particles can scatter directly off the horizon with amplitude of order 1 . To see this, note the computation is

$$
\left\langle 0\left|a_{\text {late }} a_{\text {late }}^{\dagger}\right| 0\right\rangle=1+\frac{1}{e^{\omega / k T}-1}
$$

using $a a^{\dagger}-a^{\dagger} a=1$ and (4.1), with $|0\rangle$ the initial infalling vacuum state. 


\section{Conclusions}

Without a cutoff, a massless field in a black hole background has modes with very low frequency, when measured via the timelike Killing vector, but very short wavelength, that potentially can carry information about the black hole initial state to late time interior observers. This process is incompatible with the black hole complementarity resolution of the information paradox.

In this paper we have explored a lattice model containing features expected in an effective description derived from a more fundamental holographic theory, such as AdS/CFT. The cutoff eliminates these dangerous modes, and introduces a number of new features which we have explored. We conclude that quantum information that enters the black hole horizon is erased in a scrambling time from the viewpoint of interior observers. This supports one of the key conjectures of the proposal of [1] for building consistent interior observables within a framework that solves the information puzzle.

\section{Acknowledgments}

The research of D.L. was supported in part by DOE grant DE-SC0010010 and an FQXi grant. The research of L.T. was supported in part by Icelandic Research Fund grant 130131-053, the University of Iceland Research Fund, and the Swedish Research Council under contract 621-2014-5838.

Open Access. This article is distributed under the terms of the Creative Commons Attribution License (CC-BY 4.0), which permits any use, distribution and reproduction in any medium, provided the original author(s) and source are credited.

\section{References}

[1] D.A. Lowe and L. Thorlacius, Black hole complementarity: the inside view, Phys. Lett. B 737 (2014) 320 [arXiv: 1402.4545] [INSPIRE].

[2] S. Corley and T. Jacobson, Lattice black holes, Phys. Rev. D 57 (1998) 6269 [hep-th/9709166] [INSPIRE].

[3] D. Kabat, G. Lifschytz and D.A. Lowe, Constructing local bulk observables in interacting AdS/CFT, Phys. Rev. D 83 (2011) 106009 [arXiv:1102.2910] [InSPIRE].

[4] D.A. Lowe and L. Thorlacius, Comments on the black hole information problem, Phys. Rev. D 73 (2006) 104027 [hep-th/0601059] [INSPIRE].

[5] S.L. Braunstein, S. Pirandola and K. Życzkowski, Better late than never: information retrieval from black holes, Phys. Rev. Lett. 110 (2013) 101301.

[6] A. Almheiri, D. Marolf, J. Polchinski and J. Sully, Black holes: complementarity or firewalls?, JHEP 02 (2013) 062 [arXiv:1207.3123] [INSPIRE].

[7] S. Weinberg, Lectures on quantum mechanics, Cambridge University Press, Cambridge U.K. (2012).

[8] P. Hayden and J. Preskill, Black holes as mirrors: quantum information in random subsystems, JHEP 09 (2007) 120 [arXiv:0708.4025] [INSPIRE]. 
[9] L. Susskind and L. Thorlacius, Gedanken experiments involving black holes, Phys. Rev. D 49 (1994) 966 [hep-th/9308100] [INSPIRE].

[10] L. Brillouin, Wave propagation and group velocity, Academic Press, U.S.A. (1960).

[11] S.B. Giddings, Nonviolent information transfer from black holes: a field theory parametrization, Phys. Rev. D 88 (2013) 024018 [arXiv: 1302.2613] [INSPIRE].

[12] E. Farhi, A.H. Guth and J. Guven, Is it possible to create a universe in the laboratory by quantum tunneling?, Nucl. Phys. B 339 (1990) 417 [INSPIRE].

[13] G.L. Alberghi, D.A. Lowe and M. Trodden, Charged false vacuum bubbles and the AdS/CFT correspondence, JHEP 07 (1999) 020 [hep-th/9906047] [INSPIRE].

[14] D.A. Lowe and S. Roy, Punctuated eternal inflation via AdS/CFT, Phys. Rev. D 82 (2010) 063508 [arXiv: 1004.1402] [INSPIRE].

[15] S. Corley and T. Jacobson, Hawking spectrum and high frequency dispersion, Phys. Rev. D 54 (1996) 1568 [hep-th/9601073] [INSPIRE].

[16] D. Marolf and J. Polchinski, Gauge/gravity duality and the black hole interior, Phys. Rev. Lett. 111 (2013) 171301 [arXiv: 1307.4706] [INSPIRE].

[17] S.G. Avery and D.A. Lowe, Event horizons and holography, arXiv:1310.7999 [INSPIRE].

[18] S.G. Avery and D.A. Lowe, Typical event horizons in AdS/CFT, arXiv:1501.05573 [INSPIRE]. 